\title{
LA FUNCION PUBLICA VIGENTE EN FRANCIA
}

\author{
Por \\ Emilio Casado Iglesias
}

SUMARIO: 1.- EL ESTATUTO DE 4 DE FEBRERO DE 1959; PRECEDENTES Y CONTENido general. 2.- Criticas al Estatuto. 3.- El Vigente Estatuto de LA funCION PUBLICA DE 11 DE ENERO DE 1984. 4.- ADQUISICION Y CONDICIONES DE LA NATURALEZA DE FUNCIONARIO. 5.- DERECHOS Y DEBERES DE LOS FUNCIONARIOS. LA POTESTAD DISCIPLINARIA. 6.- LA PÉRDIDA DE LA CUALIDAD DE FUNCIONARIO. 7.Los organos VIGENTES de LA FUNCION PUBLICA. 8.- LA ECOLE NATIONAL DE L'ADMINISTRATION Y LA DEMOCRATIZACION Y DESCENTRALIZACION DE LA FUNCION PUBLICA.

1. EL ESTATUTO U ORDENANZA DE 4 DE FEBRERO DE 1959.- PRECEDENTES Y CONTENIDO GENERAL.

Como dice Debbassch (1) el término funcionario tiene en Francía una acepción restringida. No se aplica más que a algunos aspectos humanos de la Administración.

En efecto, para la ordenanzas de febrero de 1959 mucha parte del personal administrativo se encontraba colocado bajo un régimen de Derecho privado; de otra parte no todos los agentes públicos eran funcionarios.

Por ello, la jurisprudencia del Estado (ârret. Vigton, de 4 de junio de 1954) definió a los funcionarios como aquellos agentes públicos que participan de modo directo en la ejecución de un servicio administrativo.

En el Estatuto citado de febrero de 1959, se consideraban funcionarios a los agentes que requirieran las dos condiciones de: a) Titulación adecuada y b) Empleo permanente.

Por ello no se consideraban funcionarios en el mismo, al personal interino o temporero, a los pasantes ni a los empleados o agentes de los servicios con carácter industrial o comercial.

Pero el Estatuto de 1959 era sólo aplicable a los funcionarios de la Administración Central, los funcionarios de las entidades locales (entidades y provincias) estaban sometidos al Estatuto de abril de 1952.

Esta dualidad de los Estatutos de 1959 y el de las entidades locales no significaba, empero, descentralización funcional alguna ya que

(1) DEBBASSCH, Ch. : Ciencia administrativa, Madrid- 1981, pág. 331. 
en toda la distribución administrativa del pais tenían un representante directo los diversos Ministerios de la Administración Central.

\section{A. Como derechos fundamentales de los funcionarios que en este Estatuto, actualmente derogado, de abril de 1959, han de citarse:}

a) En primer lugar el derecho a la retribución solamente se tenía en caso de haber ejercido las funciones correspondientes. En este sueldo se comprendian indemnizaciones especiales, como eran las de familia, y las de los menores de 3 años.

El sueldo de las mujeres era igual al de los varones, y de acuerdo con el grado que ostentara el funcionario. La escala iba del 1 al $7,5 \%$ en los empleos de inferior a superior.

b) Como segundo derecho ha de citarse la jubilación. Todo funcionario que al llegar a los 60 años de edad que tuviera unos servicios de 30 años en los empleos sedentarios, o 50 años de edad y 25 de servicios para los empleados activos, tenia derecho a una pensión que es contrapartida al descuento de la parte del sueldo durante el periodo activo; la pensión tenía como base el último sueldo percibido por el funcionario.

c) Derogado el Estatuto de 1952, para los entidades locales, los Departamentos y las regiones regía el Estatuto de 10 de julio de 1964.

d) No había derecho de traspaso de unas escalas a otras en las cuatro categorias en que estaban divididos los grados de la Administración Central.

e) Finalmente ha de citarse el derecho a la huelga. Con anterioridad al año 1946, a los funcionarios les estaba prohibido el derecho a la sindicación, y por consecuencia el derecho a la huelga.

Reconocido este derecho a todos los ciudadanos por la Constitución de 1946, este derecho a la huelga le fue aplicado al funcionario.

Ello, no obstante, por razones de continuidad en el servicio el Juez competente, podía restringir ese derecho a la huelga si era abusivo o contrario a las necesidades del servicio público.

\section{B. Como obligaciones esenciales de los funcionarios, he de citar:}

a) Como primera obligación de los mismos, en la ordenanza de 1959 , la no libertad de poder emitir opiniones políticas en el ejercicio de sus funciones. 
En el servicio el funcionario francés debía mantener una estricta neutralidad política, asi como no manifestar sus opiniones politicas de ninguna clase o hacer proselitismo.

En este sentido, verbigracia, se manifestó por el Consejo de Estado (Arrêt Maquin, de 20 de febrero de 1952), indicando que fuera de las horas de servicio un policia que esta poniendo carteles en contra del Cuerpo, falta a sus obligaciones de reserva.

d) Como segunda obligación del funcionario he de citar el régimen disciplinario a que estaba sometido. Para este Estatuto de 1959, era falta disciplinaria "toda falta cometida por un funcionario en el ejercicio o con ocasión del ejercicio de sus funciones".

Era superior jerárquico el funcionario encargado de formular o instruir el expediente para la sanción correspondiente a la falta disciplinaria.

Las sanciones que el Estatuto preveia, de inferior a superior eran las de: 1) Aviso, 2) Represión, 3) Cancelación en el cuadro de ascenso, 4) Reducción de la antigüedad de la escala, 5) Rebaja de nivel, 6) Separación de oficio, 7) Retrogradación, 8) Jubilación de oficio y 9) Destino.

Sanción independiente será la de suspensión temporal, en la que el funcionario era suspendido en el ejercicio de sus funciones por iniciación de un proceso criminal por sentencia definitiva en el proceso.

En el expediente disciplinario el funcionario habia de ser obligatoriamente oído con traslado al mismo de todo el expediente. El funcionario podrá poner en conocimiento de la Comisión Paritaria lo que creyera procedente a su derecho; siendo esta comunicación o traslado un derecho fundamental.

2. CRITICAS AL ESTATUTO DE 1959, La Administración pública en Francia constituye uno de los elementos esenciales del desarrollo de la sociedad.

Todo el mundo está de acuerdo dice Grozier (2) que hay que corregir la centralización administrativa y política que caracteriza desde hace tiempo la gestión propia de toda la Administración francesa. La Administración del país, para el mismo autor, tiene una repugnancia histórica con toda afirmación y responsabilidad hacia los ciudadanos; la solución sería una organización simple, menos arbitraria y que suprimiera toda la incertidumbre en la misión del funcionario.

(2) Grozier, M. y otros: Ou va l'administration française?, Paris 1978. 
Más recientemente, para el mismo autor la clase superior de los funcionarios es una verdadera élite, que sólo lucha por mantener sus privilegios (3).

Para el autor del mismo pais (Toening (4), la administración francesa está totalmente estratificada. Las separaciones entre los administradores y los administrados son realmente muy grandes. Los estratos diferentes en el seno de la función pública crea dos grupos totalmente diferentes y que se ignoran mutuamente: el personal de ejecución y los cuerpos medios y el pequeño grupo de los grandes cuerpos.

La función de dirección está ocupada por aquellos funcionarios. "Les grands corps" reclutados por aquellas escuelas como élite, dice textualmente, se constituyen en funcionarios de las garantias personales. Cada uno de ellos quiere mantener sus garantias, sus prestigios y su grado de cuerpo. Estas particularidades, sigue, hacen totalmente rígida y estratificada la Administración francesa".

Insistiendo en la misma idea Gremion (5) señala que la Administración rural está totalmente separada de la Administración urbana, y que los preceptos se preocupan de los derechos urbanos, pero pierden su condición de representantes en los distritos rurales, de la Administración Central.

Se da, así, para el mismo autor, un bache profundo en las relaciones del aparato administrativo territorial del Estado y las municipalidades.

No muy ajeno a estos criterios es el del inglés Ridley (6), que en relación con la administración inglesa, dice que la administración francesa tiene aquellos defectos de los grandes cuerpos (Consejeros de Estado, Inspectores de Hacienda, Diplomáticos), la existencia en la misma de la dualidad de sistemas de derecho especial para unos grupos y de naturaleza privada para otros, una administración excesivamente centralizada y el predominio de élites que se traduce muy señaladamente en la persona de los Prefectos.

Wons insistiendo en los mismos defectos, señala especialmente que la administración francesa es un modelo de centralización que supone la ruptura entre las bases y su pirámide, dando ello lugar a conflictos burocráticos y a una ruptura de la igualdad del modelo administrativo.

Hemos de señalar, empero, por nuestra parte, que las técnicas de la Administración en aquella época eran ya modernas en el país.

(3) Ibidem: No se cambia la Sociedad por Decreto, Paris, 1984, pág. 129.

(4) Toening, J.C.- Ou va ... citada pág. 30

(5) Rigley y Brondel, J: Public administration in France, London, 1974, pág. 32.

(6) Gremion, P. : La administration territoriale, París 1984, pág. 84. 
Un tanto imitando a los métodos norteamericanos, en el año 1968, siendo Ministro de Economía y finanzas Michel Debré mandó aplicar con carácter de generalidad los procedimientos del P.C.B. , similares a los norteamericanos del P.P.B.S. (Planning-programing-bundres-tue gestión).

También es importante señalar que por datos de la Dirección General pública del pais, los funcionarios del Estado son en el año 1989 el número de 2.500 .000 (7).

\section{EL VIGENTE ESTATUTO DE LA FUNCION PUBLICA DE 11 DE ENERO DE 1984.}

Por consecuencia de las críticas de la Ordenanza o Estatuto de 1959 ya el 30 de abril de ese mismo año se presentó ante el Parlamento un proyecto de Ley, cuyo principal autor fue Catherine sobre la reforma administrativa francesa, en el que se pretendia crear un Comisariado General bajo la autoridad del primer Ministro o del Secretario de Estado y en cuyo texto se decía:" Artículo 10: Por un periodo de cinco años a contar desde la promulgación de la presente Ley será constituido un Comisario General de la Reforma y la modernización administrativa colocada bajo la autoridad de un Comisariado General. Será creado por Decreto y bajo la dependencia del Presidente del Consejo".

En el artículo $2^{\circ}$ del mismo, se establecía "Que el Comisariado General de la Reforma y la ordenación administrativa tendría a su cargo: 1) Desarrollar y estudiar la documentación concerniente a las atribuciones, la estructura y funcionamiento del Estado. $Y$ en particular las proposiciones de reforma resultantes de los trabajos y el Comité de miembros y el rendimiento de los servicios públicos, las quejas de la Comisión económica, y el funcionamiento de los servicios públicos y privados y segundo elaborar un plan de reforma y actualización tendente a regular y simplificar la tarea administrativa, racionalizar la estructura y la organización de los servicios del Estado, perfeccionar los métodos de trabajo y equipamiento, adaptar en consecuencia la reglamentación y los procedimientos en vigor, y eventualmente promulgar un Estatuto del personal. Este plan precisará notablemente las diferencias más importantes, los principios adoptados y las etapas de su realización, la cual deberá expirar a los cinco años previstos en el artículo 1”․ (8).

(7) Datos que recogemos de DEBBASCH, $\mathrm{CH}$ : Sciencia administrative, París, 1989, pág. 478, y que corrobora AUBY, J.M.: Institutions administratives, París, 1989, pág. 498.

(8) El texto completo de este proyecto de Ley puede verse en Catherine, R: Fonction publique, II, Sirey, 1958. 
Consecuencia de esta medida fue la promulgación del vigente Estatuto de la función pública de 11 de abril de 1984, que significa un cambio, dice acertadamente Piquemal (9), sustantivo en relación con la época anterior sobre todo con la descentralización y democratización que supone en la función pública del país.

\section{ADQUISICION DE LA NATURALEZA DE FUNCIONARIOS Y CON- DICIONES.}

El concurso es el sistema normal en Francia para reclutamiento de los funcionarios. No ajeno al mismo sistema de nuestro pais, supone el examen de aptitud de los candidatos y una atribución de la autoridad jerárquica como operación preliminar para obtener los resultados.

El concurso está regulado en los arts. 19 y siguientes del vigente Estatuto, en los artículos 36 del título III y en los artículos 29 y siguientes del título VI.

Así mismo el concurso es el procedimiento normal para el reclutamiento de los funcionarios territoriales y para los de los establecimientos hospitalarios. Hay que señalar que por, relativamente reciente Ley de 13 de febrero de 1988, en los concursos que celebren las entidades territoriales (pero no en los de la Administración Central) el concursante puede establecer diálogo con el Tribunal del concurso.

El concurso tiene declarado el Tribunal Constitucional (AIDA, 19 de febrero 1963), es una de las garantías fundamentales de la función pública.

Los artículos indicados 19 y siguientes del Estatuto, el 36 del título III del mismo, y los artículos 29 y siguientes del título IV regulan el concurso en tres fases: a) Apertura del concurso, b) Establecimiento o fijación de la lista de candidatos y, c) Nombramiento de Tribunal.

La resolución del concurso ha de proceder generalmente de una orden Ministerial y ha de contener los datos más esenciales cuales son los puestos de trabajo a proveer, el número de plazas, los ejercicios a desarrollar, la fecha límite del mismo, y su publicación en el Diario Oficial.

Cada año, la Dirección general de la Administración y el Director General de la función pública, publicará el calendario general de reclutamiento para la función pública estatal.

La lista de candidatos ha de contener: 1․ La solicitud del candidato,. 2o Un extracto del acta de nacimiento que no tenga un tiempo su-

(9) PIQUEMAL, M. y BATER, G: Droits et garanties de Fonctionaires, París, 1989, pág. 478. 
perior a los tres meses de esa solicitud. $3^{\circ}$. Un certificado del solicitante acreditativo de su estado en el servicio militar. 4 Una copia auténtica de los títulos exigidos para el concurso. $5^{\circ}$ Un certificado acreditativo de los servicios prestados al Estado y 6ㅇ, Un certificado relativo a la edad cuando se exijan esos límites.

No existen actualmente certificado acreditativo de moralidad o buena conducta.

La publicación de las listas crea el derecho del candidato a concurrir al concurso, si bien la Administración cuenta con el derecho a examinar los títulos, edad y los demás datos discrecionales.

En desarrollo del Estatuto, el Decreto de 14 de marzo de 1988 da normas para el nombramiento de los Tribunales de los concursos, tanto para los de la Administración Central como los de las Administraciones regionales, y la Administración Local.

Son normas esenciales para la actuación de estos Tribunales la imparcialidad, que garantiza a los concursantes la igualdad de condiciones en su participación, y la colegialidad. Este último requisito origina como consecuencia jurídica el que los miembros del jurado son nombrados personalmente por lo cual no pueden ser sustituidos o reemplazados.

Otras normas que se contienen en los preceptos citados del Estatuto vigente relativas a los concursos son las de igualdad de los concursantes y la libertad de actuación de los Tribunales. Los candidatos o participantes son llamados a actuar por carta certificada con acuse de recibo $u$ otro medio que justifique la recepción del candidato.

Una vez elaborada la lista por el Tribunal o Jurado, éste la propone a la autoridad investida del poder de nombramiento y según el orden establecido por el mismo. La autoridad investida del poder de nombramiento puede no hacerlo, pero si lo hace ha de hacerlo según el orden de propuesta del Tribunal.

Ello no obstante lo expuesto sobre el concurso, se contienen en el artículo 16 del Título I del Estatuto determinados supuestos en que el sistema es derogado. Estos supuestos son los de a) la aplicación sobre empleos reservados, b) la constitución de un empleo para la transferencia de cuerpos, c) El reclutamiento en las categorias $C$ y $D$ de acuerdo con determinados Estatutos particulares, E) Los cargos de nombramiento del Gobierno, F) El nombramiento directo en determinados cuadros de la administración territorial, G) Determinados cambios de un establecimiento público para ocupar otro cargo en otro establecimiento, H) El caso de integración total o parcial de un funcionario en el mismo cuadro de su categoría e, I) El reclutamiento directo de la categoría $A$, o de cargos asimilados a otra categoría o jerarquia de ciertos Cuerpos del Estado o de la Administración Territorial y j) Examen profesional. 
Para la consideración de grado que consagra el Estatuto es preciso señalar que ese concepto de grado o cuerpo es "el conjunto de grados que, sin solución de continuidad, se sitúan en la jerarquía administrativa homogénea y la función y garantía de la gente, ambos de escalón hasta la totalidad de su clase".

En los artículos 29 del título II y en el 4 del título IV, se agrupa a los funcionarios del Estado en los grados de A, B, C y D,

La situación de la clasificación vigente en relación con el Estatuto de 1959 es que en el sistema anterior al vigente hablar de carrera era sinónimo que hablar de cuerpo.

En la Administración Territorial, que abarca cual se ha indicado, actualmente a los funcionarios de las comunas o municipios, de los Departamentos, de las Regiones y de los Establecimientos públicos, el artículo 118 de su Estatuto referido de 1987 señala las clases o grados de $A, C$ y $D$,

Cada cuadro de empleo reagrupa a los funcionarios sometidos a un estatuto particular o a los titulares del mismo grado.

En la rigidez o estancamiento que se daba con anterioridad al vigente Estatuto de 1984 en los cuadros administrativos, se ha paliado, si no ha desaparecido, por el actual sistema de ingresos y promoción y cambio en la función pública.

Para el ingreso en la categoría de funcionario $A$, el concurso es nacional; para los de las categorías $B, C$ y $D$, el concurso se lleva a cabo en el centro de gestión.

En el orden de la Administración territorial el ingreso en las categorías $C$ y $D$, se lleva a cabo por concurso entre las colectividades competentes para la provisión del empleo o cargo.

En los establecimientos hospitalarios el Decreto de 30 de Noviembre de 1988 establece las categorías de: A). Cuerpo de enfermeras, B) Cuerpo de enfermeras de salas de operaciones, C). Anestesistas Recuperadores y D). Cuerpo de puericultoras. En relación a estos establecimientos los artículos 32 al 36 del Estatuto hablan de "denominación de cambio de establecimiento".

La rigidez de este último cuerpo ha sido criticado por la doctrina (10), por cuanto significa una excepción a la movilidad de los demás cuerpos del Estado y de la Administración territorial.

Dentro de cada grado, y cual se ha indicado ya, de acuerdo con el párrafo 1 del artículo 1 del Estatuto cada cuerpo puede darse un Estatuto particular que revestirá para cada uno de ellos el carácter de nacional.

El grado que ocupe el funcionario, como dijo el Ministro de la función pública del país, al presentar el Proyecto de Ley al Parlamento, significa que la administración no puede cuestionar ese grado y es una garantía del funcionario en el cargo para el que ha sido nombrado. 
Para el ingreso en los grados $A, B, C$ y $D$ es preciso el título universitario. Para el ascenso dentro cada grado juega un papel mixto la titulación y los años de servicio.

Para el ingreso en la función pública territorial es preciso un diploma al menos de cuatro años de estudios superiores de acuerdo con el Decreto de 2 de marzo de 1988, que desarrolla su referido Estatuto del año 1987.

La posesión de un grado de funcionario es una de las garantías fundamentales del mismo que no puede ser modificado ni derogado por otra norma que no sea de superior rango jerárquico a la del Estatuto. Nada dice el mismo si sean precisas o necesarias determinadas condiciones familiares o sociales para la pertenencia a un grado, por lo que ha de entenderse la igualdad de todos los ciudadanos para pertenecer a cualquiera.

Indicado ya que no puede haber discriminación por razón de sexo, raza o creencias filosóficas o religiosas para la accesión al funcionariado del país, son requisitos para su posible pertenencia al mismo (sea en la Administración Central, sea en la territorial): A). Poseer la nacionalidad francesa (es de señalar al efecto la unión francesa creada por la Constitución del país del año 1946, esta unión se denomina actualmente Comunidad francesa) B) Gozar de los derechos civiles, C) La existencia de moralidad exigida por el Estatuto de 1959 ha sido sustituida en el vigente por la no incompatibilidad en el ejercicio de la función por acciones judiciales, D) Haber cumplido el servicio militar y, E) Tener la condición de aptitud física normal para el desarrollo de la función.

\section{DERECHOS Y DEBERES DE LOS FUNCIONARIOS.}

\section{A). Derechos.}

Como primer derecho del funcionario, no permitido, por cierto, en la etapa anterior, ha de señalarse el de la libre manifestación de sus ideas políticas.

La Ley citada de abril de 1987 para las entidades territoriales ha extendido este derecho a las mismas.

Como segundo derecho de los funcionarios ha de citarse su derecho a la promoción. Desarrollando el Estatuto, las leyes de 22 de Noviembre de 1985 y 19 de agosto de 1987, han declarado que este derecho lo tienen todos los funcionarios del país.

Incluido ya en el Estatuto de 1959, la promoción actualmente puede ser de las dos clases de: inicial o continua.

El perfeccionamiento inicial tiene por contenido en el Estatuto el doble fin de perfeccionamiento profesional y el desarrollo de la función 
social con la armonización de los medios de adaptación a las técnicas y a la economía moderna. Textualmente dice al efecto el artículo 22 del Estatuto que "El derecho a la formación profesional es reconocido a los funcionarios".

El derecho inicial al perfeccionamiento puede llevarse a cabo en la tradicional ENA, o en las demás escuelas de funcionarios de más reciente creación (E. N. M. , ENE o IRA) y aparte el fin indicado del perfeccionamiento de los funcionarios, to es también el de acentuar el carácter interadministrativo de los cuerpos y favorecer el paso de la función pública estatal a la territorial o viceversa.

En relación a ese paso de la Administración Central a la Territorial, la Ley de 1987 de las Administraciones Territoriales dice que esa promoción o perfeccionamiento lo hará el Centro Nacional del Servicio Público, dotado al efecto de un Consejo orientador y asistido de una Delegación Regional o interdepartamental.

La movilidad del funcionario no puede dar lugar: a) A la ruptura de la carrera del funcionario b) La movilidad no puede dar lugar a una ventaja en la carrera $y, c)$ La movilidad no puede perjudicar al funcionario (articulos 60 y siguientes).

Como tercer derecho de los funcionarios ha de citarse la remuneración, ya consagrada en los anteriores Estatutos. La remuneración viene establecida actualmente en el Título I del Estatuto, en el que se afirma textualmente "Que todo funcionario tiene derecho a una remuneración"; remuneración, que no hace falta decir, comprende a todos los funcionarios del país.

Esta remuneración económica comprende: 1) el sueldo, 2) Las indemnizaciones de residencia $y, 3$ ) Los suplementos familiares $u$ otros que se establezcan reglamentaria o legalmente.

1.- El sueldo.- El sueldo tiene un carácter impersonal y un carácter de prestación administrativa. Su cuantía viene dada en relación al grado $y$ al cargo que desempeñe el funcionario. $Y$ personal significa que en él no se tienen en cuenta característica personal alguna del funcionario; todos los funcionarios del mismo grado tienen derecho a la misma remuneración.

El sueldo tiene la doble cualidad de pensión alimenticia y compensación al grado o jerarquía que el funcionario detente y a la compensación de sus años de servicio.

Para su efectividad la Administración lleva una lista de los funcionarios y el grado que ocupan en la misma.

2.- Las indemnizaciones de residencia. Estas indemnizaciones fueron creadas para los funcionarios por primera vez en el año 1962 y adquirieron naturaleza jurídica a partir del Decreto de 24 de marzo del mismo año. 
Estas indemnizaciones por residencia vienen fijadas en el Decreto de 30 de julio de 1987, en el que se fijaron unos indices que van en una escala de 271 a 814 según el grado y categoría del funcionario.

Más recientemente, por Decreto de 4 de julio de 1989 se han fijado estas indemnizaciones de residencia para los funcionarios de las categorías $C$ y $D$, teniendo en cuenta los siguientes índices: escala I, indices de 209 al 282.

3.- El suplemento familiar.- Este suplemento familiar constituye un suplemento del sueldo y es una indemnización por los hijos menores de 3 años de edad. Esta indemnización o suplemento familiar viene prevista y regulada por el título II del libro V del Código de la Seguridad Social.

En efecto, en este sentido tiene declarado el Consejo de Estado (Arret de 22 de mayo de 1955) que "Las jurisdicciones especiales de la Seguridad Social son las competentes para conocer de los litigios entre el régimen especial de la Seguridad Social de los funcionarios y las autoridades administrativas",

En caso de divorcio o separación matrimonial, es el encargado de la tutela del menor el que cobrará la correspondiente ayuda.

4.- Otro derecho del funcionario es el de su elegibilidad para los cargos políticos. Con algunas exclusiones determinadas, principalmente por la jerarquia del cargo administrativo, el funcionario que fuere elegido para un puesto político conserva durante el mismo su derecho al sueldo y su derecho al ascenso.

\section{B). Deberes.}

Como correspondencias a las retribuciones económicas, el funcionario tiene marcados expresamente determinados deberes.

Estos deberes pueden clasificarse en las dos clases de deberes durante el ejercicio del cargo y los posteriores a él.

Como deberes fundamentales durante el desempeño del cargo he de enumerar:

10 El deber de residencia.

2- La obligación de dignidad, prohibiéndole, así, al funcionario dar lugar a escándalos o llevar a cabo actos que hagan su situación difícil e incompatible con el cumplimiento del deber del servicio.

30 La imparcialidad política, es decir la obligación de suministrar al público todas las informaciones que le sean pedidas en relación al servicio, salvo el secreto profesional. 
$4^{\circ}$ La de ejercer sus funciones; obligación que lleva consigo la de ejercer el servicio de forma continua y ocupar el empleo.

En relación al horario actualmente vigente, le vienen fijados al funcionario francés en 39 horas semanales, y a los funcionarios subalternos en 49 y media horas.

$5^{\circ}$ El ejercer exclusivamente la función. Como dice el art. 25 de la Ley de julio de 1983 el funcionario debe estar consagrado al ejercicio de la función que le esté encomendada.

$6^{\circ}$ La prohibición de la compatibilidad de la función pública con un empleo privado. De acuerdo con el mismo artículo 25 citado del Estatuto "Está prohibido a todo funcionario ejercer una actividad lucrativa de la naturaleza que sea".

$7^{\circ}$ El deber de obediencia. De acuerdo con el artículo 23 de la repetida Ley de julio de 1983, el funcionario tiene el deber de respetar el Estatuto y los Reglamentos correspondientes, así como las órdenes de los superiores.

En relación a ésta última obligación la jurisprudencia, no obstante, tiene declarado (Arrêt Langenod Mille Fremont, de 3 de Noviembre de 1967) la exención del deber de obediencia jerárquica si la orden del superior es ilegalmente grave y flagrante, y

$8^{\circ}$ La moralidad profesional. Esta obligación lleva consigo la prohibición de recibir remuneraciones por el servicio, la imparcialidad y la neutralidad, el secreto profesional y la discreción, y la responsabilidad penal por actos del funcionario incluidos en ese ordenamiento.

\section{LA POTESTAD DISCIPLINARIA.}

El régimen disciplinario está incluido en el vigente ordenamiento de la función pública del país como "es falta disciplinaria toda falta cometida por el funcionario en el ejercicio u ocasión de sus funciones". El superior jerárquico del funcionario es el encargado de formar el expediente para la sanción correspondiente por la falta disciplinaria.

Las sanciones que enumera el vigente Estatuto de 1984 son las de:1) Aviso, 2) Reprensión, 3) Cancelación del cuadro de ascenso, 4) Reducción de la antigüedad en la escala, 5) La rebaja de nivel, 6) Separación del servicio, 7) Retrogradación, 8) Dilación de oficio y, 9) Despido.

Sanción independiente es la de suspensión temporal en la que el funcionario es suspendido en el ejercicio de sus funciones por iniciación de un proceso criminal hasta el final por sentencia definitiva del juicio. 
En el expediente disciplinario, el funcionario ha de ser obligatoriamente oído con traslado al mismo del expediente. El funcionario pondrá en conocimiento de la Comisión Paritaria encargada de informar sobre la sanción correspondiente, lo que crea conveniente a su defensa. Este derecho de posible comunicación está declarado como uno de los derechos fundamentales de la función pública.

\section{LA PERDIDA DE LA CUALIDAD DE FUNCIONARIOS.}

Con no mucha diferencia de las causas enumeradas en la anterior ordenanza de 1959, el vigente Estatuto señala como causas de ruptura de la relación funcionarial con la administración, en artículo 24 , las de: a) La renuncia al cargo, b) La dimisión del funcionario debidamente aceptada, 3) El licenciamiento y 4) La revocación.

\section{ORGANOS VIGENTES DE LA FUNCION PUBLICA .}

La Presidencia de la organización pública francesa corresponde al Primer Ministro, el cual tiene la competencia de los fines estatales y la potestad reglamentaria.

Esa presidencia, sin embargo, es más bien teórica, por cuanto, generalmente, el Presidente delega en algún Ministro o en algún Secretario de Estado dichas competencias.

Organo máximo de esa función pública es el Ministro de la Función Pública, que bien por si mismo, o a través de la Dirección General de la Función pública ejerce las funciones correspondientes.

Creado este último organismo citado en el año 1946, son ella, con el Ministro de Hacienda los encargados de todo lo relativo al personal funcionario y a los créditos habilitados para el pago de los mismos.

En el orden inferior o de las corporaciones locales, el Ministerio del Interior dispone de la Dirección General de Corporaciones locales, que es la encargada de las relaciones o ensamblaje de estas funciones pública de las diversas Administraciones Territoriales.

La Dirección General hospitalaria elabora los planes y es la encargada de esta específica función.

Modificado el Estatuto de 1984 a estos efectos por la Ley de 22 de septiembre de 1985 y el Decreto 643, de 25 de junio del mismo año, estos han creado los Centros de gestión de la función pública Territorial.

El Consejo Superior de la Función Pública es el órgano máximo consultivo de la misma; está presidido por el Ministro de la Función Pública y está compuesto por un número igual de representantes de 
los funcionarios, de organismos sindicales, y representantes de la Administración.

En el orden inferior el Consejo Superior de la Función Pública Territorial, por el mismo carácter de órgano consultivo, ha sido creado por el Estatuto de 1984; y se compone de representantes de los organismos sindicales de la función territorial y de los funcionarios de las distintas categorias de las colectividades locales.

El Consejo Superior de la Función pública ha de ser consultado en todo proyecto de Ley o Decreto que sea de interés al funcionariado, y dé unidad, por otra, a todos los funcionarios.

Finalmente, por la Ley de 13 de julio de 1987 ha sido suprimido el organismo creado con anterioridad para esta Administración Territorial, que constituía una Comisión Mixta de representantes y funcionarios de la Administración territorial y la de las Corporaciones Locales.

\section{L'ECOLE NATIONAL DE L'ADMINISTRATION Y LA DESCENTRALI- ZACION Y DEMOCRATIZACION DE LA FUNCION PUBLICA.-}

Por la Ley de 19 de enero de 1983 han sido derogados los Estatutos de la ya tradicional y famosa escuela de Administración Pública francesa (ENA). Los nuevos Estatutos de referida Escuela, que con la Politécnica fueron tradicionalmente las dos Escuelas de la élite o jerarquía del funcionariado francés, han sido equiparados, con fecha 23 de diciembre del año 1986, para la entrada en ellas a las de otras escuelas superiores (ENN, ENC o IRA citadas).

Esta igualdad de acceso al funcionariado de todos los candidatos con el derecho a movilidad de los funcionarios en las distintas categorías, ya citado, a la función pública del país quita toda critica de centralismo o elitismo clasista.

Cual se ha indicado, la movilidad es uno de los derechos de todos los funcionarios del país, restringido solamente para los de los Establecimientos hospitalarios. Esta movilidad o paso del funcionario puede ser (Decreto de 14 de junio para el funcionariado estatal y Ley de 12 de julio para el de las Administraciones ) previo por un examen profesional. Quedan, no obstante, impedidos de ese derecho general de paso de escala; 1. Los agentes no titulares del Estado 2. Los cuerpos de Agentes de oficina, 3. Los cuerpos de Agentes técnicos de oficina. 4. Los cuerpos de estenolaestilógrafos. 5. Los agentes notariales, 6 . Los cambios entre las categoría $A$ y $B$, a los que hay que hacerse por concurso y, 7. Los Agentes no titulares de la Administración Central y de las Entidades territoriales cuando accedan a las categorías $C$ y $D$. 
Junto a estas medidas especificamente referidas a la Función pública, la llegada del partido socialista al poder ha traido en Francia otras medidas descentralizadoras, cuales son, verbigracia, la Ley de 22 de Marzo de 1982 de "reparto de competencias entre los municipios, los Departamentos y la regiones". En este sentido se dice en el arto 1 을 de citada Ley que "Las leyes determinarán las repartición de competencia entre los municipios, los departamentos, las Regiones y el Estado".

Esta Ley de 1982 ha sido desarrollada por el Decreto 83 de 7 de Enero de 1983, en cuyo articulo 5o se determina que "Las competencias pertenecientes al Estado se traspasan a los municipios, los Departamentos y las Regiones en la medida que sean necesarias para la eficacia normal de sus competencias". Corroborando estos principios el párrafo 1 del art. 3 del mismo Decreto establece que "Los municipios, los Departamentos y las Regiones constituyen el cuadro institucional de la participación ciudadana en la vida local y garantizan la expansión de su diversidad". Estas medidas , como dice acertadamente Moderne (10), es claro índice de la actual descentralización francesa y el que los principios de transferencia no deben perjudicar a las corporaciones locales.

Comentando las mismas medidas el autor del mismo país Cotten dice que, ahora, por la Ley de 1982, se han llevado a cabo cuatro medidas efectivas: a) El repartimiento de los diversos poderes, b) Suprimir la tutela sobre los entes inferiores y transferir poderes de ejecución de los Prefectos a los Consejos Generales y competencias para la elección de ese cargo. b) Un reparto de competencias, entre el Estado y las entidades territoriales, lucha a la que se venia asistiendo desde 1986, comprendiendose, también en esta descentralización medidas sobre urbanismo, asistencia social, medidas escolares y de formación profesional, c) la promulgación de un nuevo estatuto sobre la función pública $y, d$ ) tratamiento y adecuado reparto en las haciendas estatales y locales (11).

Medida también descentralizadora es la Ley de 30 de mayo de 1985 que deroga el anterior derecho común y le concede a los Departamentos y municipios el desarrollo económico, social y cultural de la montaña.

(10) Moderne, F; La répartition de competences, en volumen colectivo "Nouvelles competences locales", Paris 1985, pág. 6.

(11) Así, en relación a este tema le han sido traspasados a los Municipios las clases de tasas o impuestos de matriculación de vehículos automóviles (Carter Pris) tasas sobre los vehículos a motor (Viguettes) y los derechos de tasas de publicidad de Apartamentos (Douce, J.C: Fiscalité et décentralitation, en volumen colect. "Les nouvelles ... citado, pág. 77). 
Este desarrollo comprende, más concretamente, actividades agropecuarias y forestales, desarrollo turístico, cuencas, transportes necesarios para el turismo y estaciones, actividades comerciales y artesanas, seguridad pública y protección social de los riesgos de montaña, disposiciones financieras ligadas a esa actividad, recursos hidráulicos y protección del medio ambiente, asi como normas de urbanismo y de transporte mecánico. 